\title{
Nonlinear Optical Characterization of GaN Layers Grown by MOCVD on Sapphire
}

Ivan M. Tiginyanu, Igor V. Kravetsky, Dimitris Pavlidis ${ }^{1}$, Andreas Eisenbach ${ }^{1}$, Ralf Hildebrandt ${ }^{2}$, Gerd Marowsky ${ }^{2}$, Hans L. Hartnagel ${ }^{3}$

Laboratory of Low-Dimensional Semiconductor Structures, Institute of Applied Physics, Technical University of Moldova, 2004 Chisinau, Moldova, tiginyanu@mail.md

${ }^{1}$ Solid State Electronics Laboratory, Department of Electrical Engineering and Computer Science, University of Michigan, 1301 Beal Avenue, Ann Arbor, MI 48109-2122, U.S.A.

${ }^{2}$ Laser-Laboratorium Göttingen, Hans-Adolf-Krebs-Weg 1, D-37077 Göttingen,

Germany

${ }^{3}$ Institut für Hochfrequenztechnik, Technische Universität Darmstadt, D-64283

Darmstadt, Germany

\section{ABSTRACT}

Optical second and third harmonic generation measurements were carried out on GaN layers grown by metalorganic chemical vapor deposition (MOCVD) on sapphire substrates. The measured $d_{33}$ is 33 times the $d_{11}$ of quartz. The angular dependence of second-harmonic intensity as well as the measured ratios $\mathrm{d}_{33} / \mathrm{d}_{15}=-2.02$ and $\mathrm{d}_{33} / \mathrm{d}_{31}=$ -2.03 confirm the wurzite structure of the studied GaN layers with the optical c-axis oriented perpendicular to the sample surface. Fine oscillations were observed in the measured second and third harmonic angular dependencies. A simple model based on the interference of the fundamental beam in the sample was used to explain these oscillations.

\section{INTRODUCTION}

Recently there has been strong interest in studying gallium nitride and alloys because of their promising device applications [1]. In particular, the nonlinear optical properties of $\mathrm{GaN}$ films are of interest for optoelectronic and all-optical device applications. Several second-harmonic generation (SHG) and third-harmonic generation (THG) studies of GaN epilayers have been reported recently [2-5]. However, no attention was paid to the differences between front and back excitations nor to the angular step resolution on the nonlinear optical response. In the present work we report on the study of nonlinear optical effects in $\mathrm{GaN} /$ sapphire samples as a function of excitation geometry and angular step resolution. Both the second-harmonic ( $\mathrm{SH})$ and third-harmonic $(\mathrm{TH})$ signals measured in dependence on the incident angle of the laser beam were found to be modulated due to the interference of the fundamental beam in the sample. 


\section{EXPERIMENT}

The GaN layers used in our experiments were grown by low-pressure MOCVD on (0001) c-plane sapphire using trimethylgallium (TMGa) and ammonia $\left(\mathrm{NH}_{3}\right)$ as source materials. A horizontal growth reactor in a modified EMCORE GS-3200 system was used for this purpose. A buffer layer of about $25 \mathrm{~nm}$ thick GaN was first grown at $510{ }^{\circ} \mathrm{C}$. The GaN layers grown on top at a temperature of $1100{ }^{\circ} \mathrm{C}$ had thicknesses of about $1 \mu \mathrm{m}$.

Polarized SHG and THG measurements were carried out in transmission mode for both front (the GaN layer facing the incident pump beam) and back (the sapphire substrate facing the incident pump beam) excitations. As a fundamental beam, the 1064 nm output of a Q-switched Nd-YAG laser (Spectra Physics GCR-170) with $10 \mathrm{~Hz}$ repetition rate and $7 \mathrm{~ns}$ pulse width was used. To minimize the influence of the laser output fluctuations, the measured SH ( $\left.\lambda_{2 \omega}=532 \mathrm{~nm}\right)$ and TH $\left(\lambda_{3 \omega}=355 \mathrm{~nm}\right)$ intensities were normalized by the simultaneously monitored laser intensity in the reference channel. The sample was mounted on a step-motorized rotation stage. The direction of the fundamental beam polarization was changed by rotating a half-wave plate placed in front of the sample. The fundamental wavelength was filtered out from SHG and THG signals by using appropriate colour filters and a grating monochromator.

\section{RESULTS AND DISCUSSION}

The wurzite structure of GaN belongs to the $6 \mathrm{~mm}$ point group symmetry. In this case there are three nonzero nonlinear optical coefficients $d_{15}, d_{31}$, and $d_{33}$, which are responsible for the second-order nonlinear optical properties. The induced nonlinear polarization in $\mathrm{GaN}$ films has the following components [6]:

$$
\begin{aligned}
& P_{x}(2 \omega)=2 d_{15} E_{z}(\omega) E_{x}(\omega) \\
& P_{y}(2 \omega)=2 d_{15} E_{z}(\omega) E_{y}(\omega) \\
& P_{z}(2 \omega)=d_{31}\left(E_{x}(\omega)^{2}+E_{y}(\omega)^{2}\right)+d_{33} E_{z}(\omega)^{2}
\end{aligned}
$$

According to equations (1), SHG is forbidden from a c-textured film (having the optical axis c perpendicular to the surface) when the pump beam is incident normal to the film. One can notice that for an ideal wurzite structure, the nonzero elements of the second-order nonlinear susceptibility tensor are related to each other as $\mathrm{d}_{15} \approx \mathrm{d}_{31}$ and $\mathrm{d}_{33} / \mathrm{d}_{31} \approx-2[7,8]$.

Neglecting absorption and birefringence of $\operatorname{GaN}\left(\Delta \mathrm{n}_{\omega(2 \omega)}=0.02\right)[9]$, the transmitted SHG intensity $I_{2 \omega}$ as a function of the incident angle $\theta$ of the fundamental beam can be written as follows $[10,11]$ :

$$
\mathrm{I}_{2 \omega}^{\mathrm{m}-\mathrm{p}}(\theta) \propto C \mathrm{I}_{\omega}{ }^{2}(2 \pi \mathrm{L} / \lambda)^{2}\left(\mathrm{~d}_{\mathrm{eff}}^{\mathrm{m}-\mathrm{p}}\right)^{2} \sin ^{2} \Psi / \Psi^{2}
$$

Where $\mathrm{I}_{2 \omega}{ }^{\mathrm{m}-\mathrm{p}}$ is the p-polarized SH intensity induced by the m-polarized fundamental beam (i.e., s- or p-polarized); $\mathrm{C}$ is a parameter determined by the appropriate Fresnel transmission coefficients and the beam area; $L$ is the layer thickness; $\Psi(\theta)=(2 \pi L / \lambda)\left(n_{\omega}\right.$ 
$\left.\cos \theta_{\omega}-\mathrm{n}_{2 \omega} \cos \theta_{2 \omega}\right)$, where $\mathrm{n}_{\omega}$ and $\mathrm{n}_{2 \omega}$ are the refractive indices at the fundamental and SH frequencies in the layer; $\theta_{\omega}$ and $\theta_{2 \omega}$ are the refractive angles of fundamental and SH waves determined by $\sin \theta=\mathrm{n}_{\omega} \sin \theta_{\omega}$ and $\sin \theta=\mathrm{n}_{2 \omega} \sin \theta_{2 \omega}$, respectively; $\mathrm{d}_{\text {eff }}^{\text {m-p }}$ are the effective second-order nonlinear optical coefficients for appropriate polarization combinations. For the transmitted SH intensity the effective second-order nonlinear optical coefficients are:

$$
\begin{aligned}
& d_{\text {eff }}^{\text {s-p }}=d_{31} \sin \theta_{2 \omega} \\
& d_{\text {eff }}^{45-p}=d_{15} \sin \theta_{\omega} \\
& d_{\text {eff }}^{p-p}=d_{15} \cos \theta_{2 \omega} \sin 2 \theta_{\omega}+d_{31} \cos ^{2}\left(\theta_{\omega}\right) \sin \theta_{2 \omega}+d_{33} \sin ^{2}\left(\theta_{\omega}\right) \sin \theta_{2 \omega}
\end{aligned}
$$

The coefficients $\mathrm{d}_{15}, \mathrm{~d}_{31}$, and $\mathrm{d}_{33}$ can be determined by measuring the $\mathrm{SH}$ intensity as a function of the incident angle of the fundamental beam for the above mentioned polarization combinations and comparing it to the $\mathrm{SH}$ intensity of a reference quartz plate.

Figure 1 shows the intensity of the transmitted p-polarized SH signal $\left(\lambda_{2 \omega}=532\right.$ $\mathrm{nm}$ ) from a $1 \mu \mathrm{m}$ thick GaN layer as a function of the incident angle of $\mathrm{p}$ - and s-polarized fundamental beam for the back excitation. The measurements were carried out with an angular resolution of $1^{\circ}$ (a) and $0.1^{\circ}$ (b) per step, respectively. In figure 1(b) fine oscillations on the curve are clearly seen. Similar oscillations were also observed in SH angular
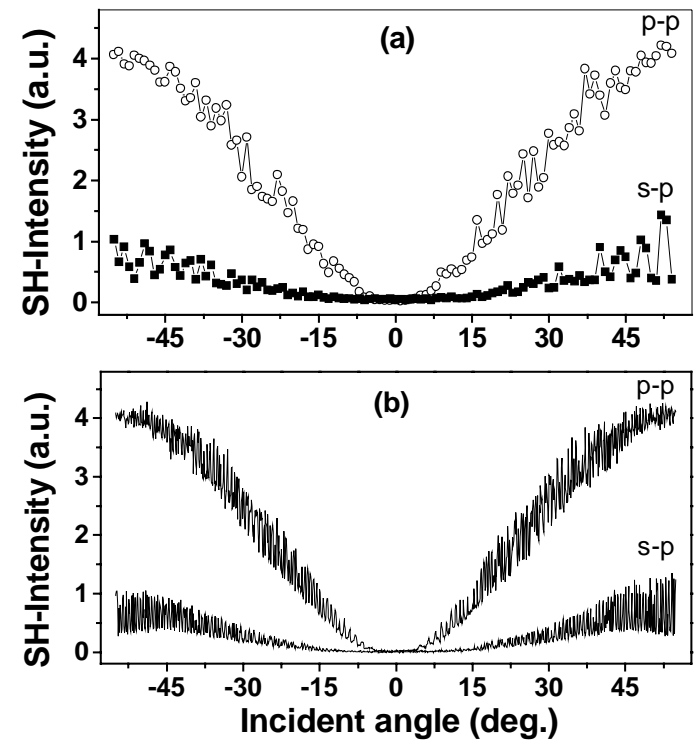

Figure 1. Measured p-polarized second-harmonic intensity as a function of the incident angle of the $p$ - and s-polarized fundamental beam. The angular resolutions are $1^{\circ}(a)$ and $0.1^{\circ}(b)$ per step, respectively. 
dependencies measured for front excitation. The angular dependence of the p-polarized second-harmonic intensity measured for the back excitation with an angular step of $0.01^{\circ}$ shows the modulated pattern of the SH-signal in more detail (see figure 2).

The obtained results show that the fine oscillations can be resolved only with an angular step of $0.1^{\circ}$ or smaller. It is of interest to note that the observation of the fine oscillations made it possible to reveal the phase shift of $\pi$ between the measured angular dependencies for the front and the back excitations.

Assuming that the sample possesses two strictly parallel faces (i.e., the sample acts as an interferometer) and taking into account only interference of the first two transmitted fundamental beams [12] we obtain in first approximation good fits (dotted line) to the experimental data depicted in figure 2. A good fit was obtained with $\mathrm{I}_{2}(\omega)=0.007 \mathrm{I}_{1}(\omega)$, where $I_{1}(\omega)$ is the intensity of the incomming fundamental beam and $I_{2}(\omega)$ is the intensity of the second transmitted fundamental beam after two reflections inside the sample.

The observed phase shift of $\pi$ between two excitations is more clearly seen by measuring the angular dependence of TH intensity, because THG is allowed at normal incidence. Figure 3 shows the intensity of the transmitted p-polarized TH intensity $\left(\lambda_{3 \omega}=\right.$ $355 \mathrm{~nm}$ ) as a function of the incident angle of the p-polarized fundamental beam for both back and front excitations. Good fits (solid lines) were obtained using the same model of the interference of two fundamental beams in the sample that was applied for the SH angular dependencies. It should be noted that neither a SH nor a TH signal from sapphire substrate was detected under the experimental conditions.

By comparing figures 2 and 3, one can see the equal number of oscillations for both SHG and THG responses. This and the presented fits suggest that the observed modulation of the nonlinear optical signal is caused by the interference of the fundamental beam in the sample. In our opinion, the observed oscillations are also indicative of a high quality of the sample.

Comparing the measured SH intensities for s-p and $45^{\circ}$-p polarization combinations (see equations 3 and 4 ) with SH Maker fringes of a reference z-cut quartz plate $\left(\mathrm{d}_{11}(2 \omega)=0.335 \mathrm{pm} / \mathrm{V}\right)$ [13], the coefficients $\mathrm{d}_{15}=5.48 \mathrm{pm} / \mathrm{v}$ and $\mathrm{d}_{31}=5.46 \mathrm{pm} / \mathrm{V}$ were deduced. The values of $d_{15}$ and $d_{31}$ relative to $d_{33}$ were found by substituting the obtained values of $\mathrm{d}_{15}$ and $\mathrm{d}_{31}$ in equation 5 , and making a least-squares fit of the

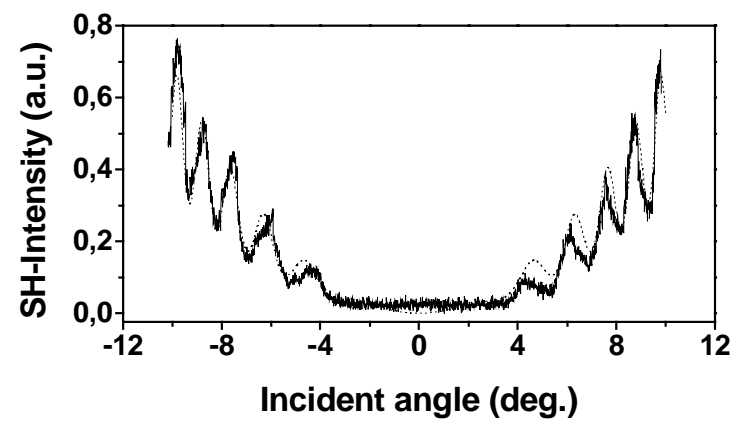

Figure 2. Measured p-polarized second-harmonic intensity as a function of the incident angle of the p-polarized fundamental beam. The angular resolution is $0.01^{\circ}$ per step. The solid line is experimental, the dotted line is a fit. 


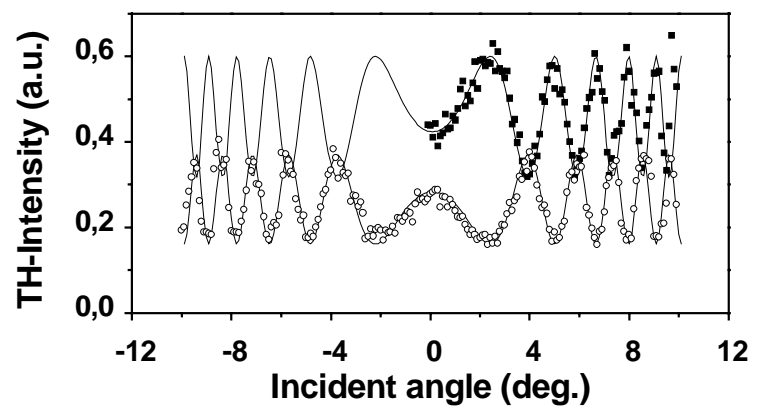

Figure 3. Measured p-polarized third-harmonic intensity as a function of the incident angle of the p-polarized fundamental beam for back (solid squares) and front (open circles) excitations, respectively. The angular resolution is $0.1^{\circ}$ per step. Solid lines show fits.

obtained angular SHG dependence for p-p polarization combination with the use of equation 2 . We found that $\mathrm{d}_{33} / \mathrm{d}_{15}=-2.02$ and $\mathrm{d}_{33} / \mathrm{d}_{31}=-2.03$, and consequently $\mathrm{d}_{33}=-$ $11.07 \mathrm{pm} / \mathrm{V}$. The obtained values are in good agreement with the ones reported in Refs. 3 and 4 .

Figure 4 shows the SH polarization dependence for front excitation at different incident angles of the pump beam. A function of the type $\left(a \cos ^{2} \varphi+b \sin ^{2} \varphi\right)^{2}$ gives a good fit to the curves indicating that $\mathrm{GaN}$ is isotropic in the plane of the film. The $\varphi$ is polarizaion angle of the fundamental beam $\left(\varphi=0\right.$ and $\varphi=90^{\circ}$ correspond to the $s$ - and $p$ polarized fundamental beam, respectively); the parameters a and $\mathrm{b}$ are determined by appropriate coefficients $\mathrm{d}$ and the transmission factors of fundamental and $\mathrm{SH}$ radiations.

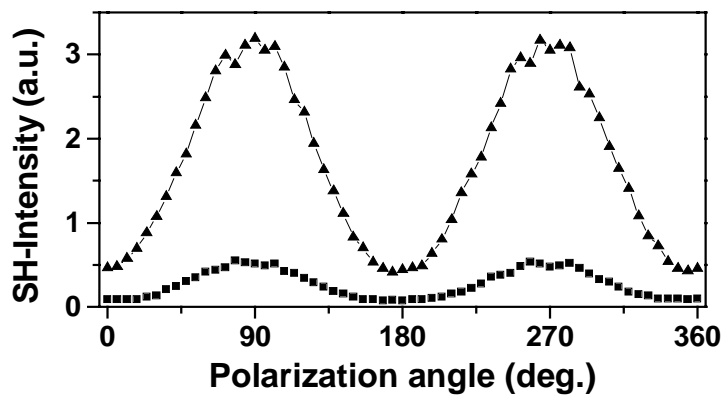

Figure 4. Measured p-polarized second-harmonic intensity as a function of polarization angle of the fundamental beam at $10^{\circ}$ (solid squares) and $30^{\circ}$ (solid triangles) incident angles of the pump beam, respectively. 


\section{CONCLUSIONS}

In conclusion, the obtained results confirmed the wurzite structure of the MOCVD GaN layers, with the optical c-axis oriented perpendicular to the sapphire substrates. The measured $d_{33}$ is 33 times $d_{11}$ of quartz. Using a high angular resolution we were able to observe fine oscillations in the nonlinear optical response of GaN. The analysis of the experimental results shows that these oscillations are caused by the interference of the fundamental beam in the sample. It is quite neccessary to take these oscillations into account by analyzing the experimental data obtained from the measurements of SHG (THG) angular dependencies. In experiments with poor angular resolution the interference pattern cannot be resolved and might be wrong interpreted as a simple scattering of the measured data. In this case, the determined values of the nonlinear optical coefficients of thin films are ambiguous.

\section{ACKNOWLEDGEMENTS}

I.M.T. and I.V.K. gratefully acknowledge the support by the Alexander von Humboldt Foundation. This work was partially supported by the NATO Scientific Division under Grant HTECH.LG 961399 and ONR contract No. N00014-92-J-1552.

\section{REFERENCES}

1. Properties, Processing and Applications of Gallium Nidride and Related Semiconductors, edited by J. H. Edgar, S. Strite, I. Akasaki, H. Amano, and C. Wetzel (EM 023, 1999), p. 680.

2. H. Y. Zhang, X.H. He, Y. H. Shih, M. Schurman, Z. C. Feng, and R. A. Stall, Appl. Phys. Lett. 69, 2953 (1996).

3. J. Miragliotta, D. K. Wickenden, T. J. Kistenmacher, and W. A. Bryden, J. Opt.Soc.Am. B 10, 1447 (1993).

4. W. E. Angerer, N. Yang, A. G. Yodh, M. A. Khan, and C. J. Sun, Phys. Rev. B 59, 2932 (1999).

5. J. Miragliotta, and D. K. Wickenden, Phys. Rev. B 50, 14960 (1994).

6. A. Yariv and P. Yeh, Optical Waves in Crystals (Wiley, New York, 1984).

7. B. F. Levine, Phys. Rev. B 7, 2600 (1973).

8. J. L. Hughes, Y. Wang, and J. E. Sipe, Phys. Rev. B 55, 13630 (1997).

9. T. Ishidate, K. Inoue, and M. Aoki, Jap. J. Appl. Phys. 19, 1641 (1980).

10. J. Jerphagon and S. K. Kurtz, J. Appl. Phys. 41, 1667 (1970).

11. W. N. Herman and L. M. Hayden, J. Opt. Soc.Am. B 12, 416 (1995).

12. M. Born and E. Wolf, Principles of Optics (Pergamon, 1964), pp. 288-353.

13. Handbook of Laser Science and Technology, edited M. J. Weber (CRC Press, Inc. Boca Raton, FL, 1986), p. 80. 